\title{
Zugriff auf Legacy-Daten in S/4HANA
}

\section{Detlev Steinbinder}

Vor dem Wechsel einer bestehenden SAP-Anwendung nach S/4HANA müssen sich Unternehmen zunächst damit auseinandersetzen, welche Migrationsstrategie am besten zu den heutigen Anforderungen oder den zukünftigen Unternehmenszielen passt. Häufig gilt es nicht nur ein einzelnes SAP-System

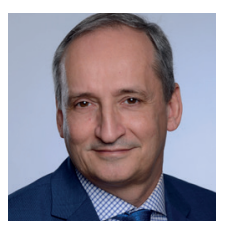

Prof. Dr. Detlev Steinbinder lehrt an der Hochschule Worms University of Applied Sciences im Fachbereich Informatik und ist Co-Founder der PBS Software $\mathrm{GmbH}$. zu migrieren, vielmehr müssen komplexe Systemlandschaften auf die neue S/4-Plattform gehoben werden. Oft wird zwischen einer klassischen Systemkonvertierung (Brownfield-Ansatz) und einem kompletten Neuaufsetzen von S/4 (Greenfield) entschieden. Bei dieser Problematik kommt dem Umgang mit den Daten der Vorgänger-Systeme eine besondere Bedeutung zu. Hierfür hat die PBS Software GmbH die mit S/4 integrierte SoftwareLösung Nearline Analytic Archive (NAA) entwickelt, die die Aufbewahrung und den Zugriff auf die Alt-Daten aus S/4 heraus gewährleistet.

$\mathrm{B}$ ei einem Greenfield-Ansatz erfolgt in der Regel ein ReDesign der SAP-Anwendung, die komplett neu aufgesetzt wird. In dem neuen System erfolgt dann eine Übernahme von wichtigen und angepassten Grund- und Stammdaten sowie offener Bewegungsdaten aus dem Altsystem. Das neue S/4-System ist somit weitgehend frei von historischen Alt-Daten. Dies gilt insbesondere für Bewegungsdaten inklusive eventuell vorhandener Archivdaten, die bei vielen Kunden sehr umfangreich sind.

Bei einem Brownfield-Ansatz wird versucht, möglichst alle Daten des Vorsystems nach $\mathrm{S} / 4$ zu migrieren, In diesem Beitrag lesen Sie:

- wie die gewählte Migrationsstrategie sich auf den Umgang mit Legacy-Daten auswirkt,

- wie in S/4HANA Legacy Daten aus ERP-Systemen übernommen und angezeigt werden können,

- wie die SAP-Datenarchivierung die Migration erleichtert. sofern es durch das neue S/4-Datenmodell und die SAP-Migrationshilfen möglich ist. Zuvor archivierte Bewegungsdaten werden aus Platz- und Performance-Gründen jedoch nicht in die neue HANA-Datenbank übernommen.

In beiden Fällen ist die Übernahme von Daten aus dem Altsystem nach S/4 unvollständig oder übernommene Daten werden durch den Migrationsvorgang an das neue Datenmodell angepasst und liegen damit nicht mehr in der ursprünglichen Form vor.

Es ist zu bedenken, dass sämtliche Alt-Daten noch jahrelang zur Abdeckung legaler und innerbetrieblicher Anforderungen für Auswertungszwecke zur Verfügung stehen müssen. Über das neue S/4-System ist dies nur mit Einschränkungen möglich und das langfristige Vorhalten der Altsysteme ist mit hohen Kosten verbunden.

Die PBS-Lösung Nearline AnalyticArchive (NAA) löst die Probleme, in dem die Alt-Daten vollständig und im Original-Format mithilfe zugehö- riger Extraktoren in eine NearlineDatenbank übernommen werden, die an das neue S/4-System angebunden ist (Side-Car-Prinzip). Der NAAExtraktionsprozess wird dabei überwacht und validiert. Somit ist eine vollständige Datenübernahme in die Nearline-Datenbank garantiert. Im Anschluss stehen das gesamte Original-Customizing und alle Stamm- und Bewegungsdaten des Alt-Systems zur Auswertungszwecke im neuen S/4-System zur Verfügung. Für die Anwender stehen erweiterte SAPStandard-Transaktionen und Reports bereit, die unter Berücksichtigung des SAP-Berechtigungskonzeptes Endbenutzer-Zugriffe auf die Alt-Daten ermöglichen. Damit erschließt sich das Potenzial, die Altsysteme nach eingehender Prüfung stillzulegen.

\section{Realisierung des Zugriffs auf Legacy-Daten in S/4HANA}

Die Alt-Daten werden durch NAATransaktionen und Reports wie in dem ursprünglichen ERP-System angezeigt, was durch die Übernahme 


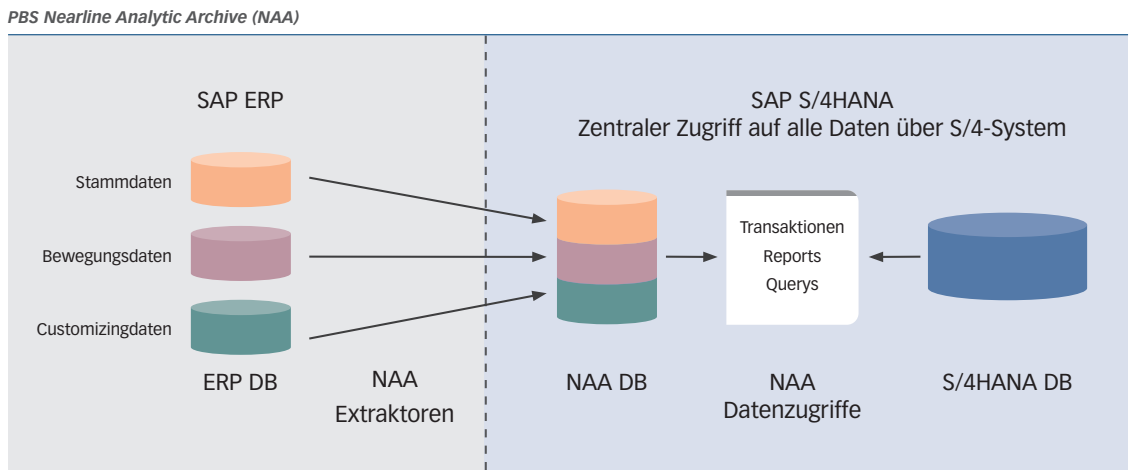

Bild 1: Architektur Datenfluss und Datenzugriff.

der Alt-Daten im Original-Format ermöglicht wird. Die Anzeigen ersetzen somit die Zugriffe aus dem Altsystem.

Darüber hinaus können sämtliche Alt-Daten im S/4-System jederzeit flexibel durch generische Querys angezeigt und ausgewertet werden. Das Anlegen der Querys ist einfach gestaltet und kann von Endbenutzern durchgeführt werden.

Zur Einschränkung des Zugriffs kann das vorhandene SAP-Berechtigungskonzept in $\mathrm{S} / 4$ genutzt werden.

\section{Kundenspezifische Daten bleiben erhalten}

Viele SAP-Systeme enthalten Modifikationen und Zusatzdaten, die üblicherweise in Z-Tabellen gespeichert werden. Mit NAA können auch diese Non-SAP-Daten in die Nearline-Datenbank übernommen werden. Neben den generischen Query-Zugriffen können kundenspezifische Auswertungen über eine ABAP-Programmierschnittstelle an die NAA-Daten jederzeit angepasst werden. Dadurch kann ein beliebiger Datenzugriff durch ABAP-Programme realisiert werden. PBS bietet hierzu entsprechende Unterstützung an.

\section{Große Datenvolumina und Performance}

Die meisten SAP-Kunden betreiben ihre ERP-Systeme seit vielen Jahren und haben über die Jahre umfangreiche Datenbestände im Terabyte-
Bereich aufgebaut. Diese Daten befinden sich in den entsprechenden SAP-Datenbanken und meist auch zusätzlich in den SAP-Datenarchiven. Bei der Übernahme in die NearlineDatenbank muss eine entsprechende Technologie vorhanden sein, die für das Datenmanagement großer Volumina geeignet ist.

PBS NAA verwendet hierzu spaltenorientierte Datenbanktechnologie von SAP, IBM oder Microsoft, mit der extrem große Datenvolumina schnell und kostengünstig verarbeitbar sind. Mit diesen skalierbaren Technologien ist NAA für kleine bis hin zu sehr großen SAP-Anwendungen einsetzbar.

\section{Konsolidierung von Mehrsystem-Landschaften}

In einigen Unternehmen werden mehrere ERP-Systeme betrieben, die in ein oder wenige S/4HANA-Systeme überführt werden sollen. Die PBS NAA-Architektur erlaubt es, Daten aus mehreren ERP-Alt-Systemen in eine physische NAA-Datenbank zu übernehmen, die an ein führendes S/4HANA-System angebunden ist. Aus dem S/4-System kann dann auf die Daten der verschiedenen AltSysteme, die logisch unterschieden werden, zugegriffen werden. Durch die Einbindung in das SAP-Berechtigungskonzept werden unberechtigte Zugriffe auf die Alt-Daten verhindert.

Die NAA-Architektur unterstützt damit direkt die Konsolidierung bestehender komplexer SAP-Systemlandschaften.

\section{Datenarchivierung}

Mit PBS NAA können ebenfalls ERPArchivdaten übernommen und in $\mathrm{S} / 4$ angezeigt werden. SAP empfiehlt, vor einer S/4HANA-Migration alle abgeschlossenen Vorgänge mithilfe der SAPDatenarchivierung aus der SAP-Datenbank zu entfernen. Dadurch wird das Volumen der SAP-Datenbank und der Umfang des zu migrierenden Datenbestandes minimiert, um die Projektlaufzeiten und Kosten zu senken. Nicht zuletzt dadurch wird der Data Footprint in der HANA-Datenbank verringert. Mit NAA gehen diese Daten in der S/4 Welt nicht verloren, sondern stehen weiterhin für Auswertungen in S/4 bereit, ohne die S/HANA Datenbank zu belasten.

\section{Schlüsselwörter:}

SAP ERP, SAP S/4HANA, Migration, Legacy-Daten, Datenarchivierung

\section{Access to legacy data in S/4HANA}

Before migrating an existing SAP ERP application to S/4HANA, companies must first consider which migration strategy best fits today's requirements or future business goals. Often it is not just a single SAP system that needs to be migrated, but complex system landscapes must be upgraded to the new $\mathrm{S} / 4$ platform. Often a decision is made between a classic system conversion (Brownfield approach) and a complete new setup of $\mathrm{S} / 4$ (Greenfield). Given this issue, the handling of the data of the predecessor systems is of particular importance. For this purpose, PBS Software $\mathrm{GmbH}$ has developed the Nearline Analytic Archive (NAA) software solution that is integrated with $S / 4$ and guarantees the storage of and access to old data from $\mathrm{S} / 4$.

Keywords

SAP ERP, SAP S/4HANA, Migration, Legacy data, Data archiving

Kontakt:

PBS Software GmbH Schwanheimer Str. 144 A

64625 Bensheim

E-Mail: info@pbs-software.com www.pbs-software.com 